Robert Mau*, Gábor Jüttner*, Ziwen Gao, Farnaz Matin, Dorian Alcacer Labrador, Felix Repp, Samuel John, Verena Scheper, Thomas Lenarz and Hermann Seitz*

\title{
Micro injection molding of individualised implants using 3D printed molds manufactured via digital light processing
}

\begin{abstract}
Here, we demonstrate a manufacturing process for individualised, small-sized implant prototypes. Our process is promising for the manufacturing of drug-releasing (micro)implants to be implanted in the round window niche (RWN-I, solid body, free-form-shaped design, 1.1 × 2.7 x 3.1 $\mathrm{mm}$ ) and for frontal neo-ostium implants (FO-I, tube-like design, length $\sim 7 \mathrm{~mm}, \varnothing \sim 2-6 \mathrm{~mm}$ ) for frontal sinus drainage. Implant prototypes are manufactured using micro injection molding $(\mu \mathrm{IM})$. We use digital light processing (DLP) as a 3D printing technique for rapid tooling of accurate molds for the $\mu \mathrm{IM}$ process. A common acrylate-based photopolymer for stiff and high-detailed modelling but with low head deflection temperature of HDT $=60.5^{\circ} \mathrm{C}$ is used for DLP 3D printing of the molds. The molds were 3D printed with a layer height of $50 \mu \mathrm{m}$ in about $20 \mathrm{~min}$ (RWN-I) and 60 min (FO-I). For $\mu$ IM investigations, we use liquid silicone rubber (LSR) as a biocompatible and medically relevant material. Micro injection molding of LSR was investigated using mold temperatures between $\mathrm{T}_{\text {mold }}=110^{\circ} \mathrm{C}$ (long $\mathrm{t}_{\text {curing }} \sim 2 \mathrm{~h}$ ) up to $\mathrm{T}_{\text {mold }}=160{ }^{\circ} \mathrm{C}$ (short $\mathrm{t}_{\text {curing }} \sim 5 \mathrm{~min}$ ).

As a result, small-sized, complex-shaped implant prototypes of LSR can be successfully manufactured via $\mu \mathrm{IM}$ using high $\mathrm{T}_{\text {mold }}=160{ }^{\circ} \mathrm{C}$ and short curing time. DLP 3D printing material with relative low $\mathrm{HDT}=60.5^{\circ} \mathrm{C}$ was
\end{abstract}

\footnotetext{
*Corresponding authors: Robert Mau ${ }^{1,2}$, Gábor Jüttner ${ }^{3}$, Hermann Seitz ${ }^{1,2}:^{1}$ Microfluidics, University of Rostock, Justusvon-Liebig-Weg 6, 18059 Rostock, Germany

${ }^{2}$ Department Life, Light \& Matter (LL\&M), Albert-Einstein-Straße 25, 18059 Rostock, Germany

${ }^{3}$ Kunststoff-Zentrum in Leipzig gGmbH, Erich-Zeigner-Allee 44 04229 Leipzig, Germany

e-mail: robert.mau@uni-rostock.de, juettner@kuz-leipzig.de, hermann.seitz@uni-rostock.de

Ziwen Gao, Farnaz Matin, Verena Scheper, Thomas Lenarz: Clinic for Oto-Rhino-Laryngology, Hannover Medical School, Hannover, Germany

Dorian Alcacer Labrador, Samuel John: HörSys GmbH, Hannover, Germany

Felix Repp: OtoJig GmbH, Hannover, Germany
}

suitable for $\mu \mathrm{IM}$. There is no significant wear of the molds, when used for a low number of $\mu \mathrm{IM}$ cycles $(\mathrm{n} \sim 8)$. Design of metal mold housing has to be suitable (perfect fit of mold, no cavities facing the molds surface for prevention of thermal expansion of mold into cavities).

Keywords: micro injection molding ( $\mu \mathrm{IM}$ ), 3D printing, digital light processing (DLP), rapid tooling, frontal neoostium implant, frontal sinus drainage, round window niche

https://doi.org/10.1515/cdbme-2021-2101

\section{Introduction}

Medical imaging methods such as cone beam computed tomography (CBCT) enable accurate, three-dimensional imaging of rigid tissue structures. For example, digital imaging and communications in medicine (DICOM) data sets can be used for digital models and exported e.g. as a standard tessellation language (STL) file for further 3D printing. Those methods hold promising chances for patient-individualised, drug-releasing implants to be implanted in the round window niche (RWN-I, Fig. 1) or for frontal sinus drainage (FO-I, Fig. 1) to offer modern therapy of inner ear disorders [1] or to challenge diseases such as chronic rhinosinusitis (CRS) [2].

To manufacture RWN-I or FO-I, accurate methods are needed to meet the implant's small size and patient-

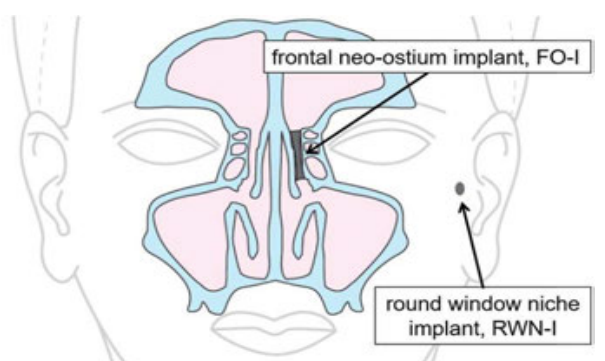

Figure 1: Location for frontal neo-ostium implant (FO-I) for frontal sinus drainage and location for round window niche implant $(\mathrm{RWN}-\mathrm{I})$ for drug-release in inner ear 
individualised, complex shape. Micro-scaled polymer parts can be manufactured with high precision via micro injection molding ( $\mu \mathrm{IM})[3]$. High-resolution 3D printing methods such as digital light processing (DLP) offer promising ways for polymer-based rapid tooling of complex molds for $\mu \mathrm{IM}[4]$. This study demonstrates the manufacturing of RWN-I and FOI prototypes via $\mu \mathrm{IM}$ utilising DLP $3 \mathrm{D}$ printing as a rapid tooling method.

\section{Materials and methods}

\subsection{Design and 3D printing of molds}

For $\mu \mathrm{IM}$ of small-sized implant prototypes of a frontal neoostium implant (FO-I) and a round window niche implant (RWN-I), individual molds for each type of implant were designed (Fig. 2). Their design was derived from the shape of the digital implant models, which are based on digital medical imaging CBCT DICOM data sets and investigated previously [1][2]. RWN-I is designed as a patient-individualised complex-shaped, solid body with a size of $1.5 \times 2.7 \times 3.4 \mathrm{~mm}$ (width $\mathrm{x}$ height $\mathrm{x}$ length). The RWN-I molds are designed as an assembly of two parts with a total size of $6 \times 7 \times 11 \mathrm{~mm}$ (width $\mathrm{x}$ height $\mathrm{x}$ length). The FO-I is designed as a tube-like structure. It has a length of $\sim 7 \mathrm{~mm}$ and the diameter varies from $\sim 2 \mathrm{~mm}$ up to $\sim 6 \mathrm{~mm}$. The mold for $\mu \mathrm{IM}$ of FO-I is designed as an assembly of seven parts with a total size of $12 \times 12 \times 30 \mathrm{~mm}$ (width $\times$ height $\times$ length).

The molds for $\mu \mathrm{IM}$ of RWN-I and FO-I were manufactured via the DLP 3D printer VIDA and a UV light curing box (both EnvisionTEC GmbH, Gladbeck, Germany).
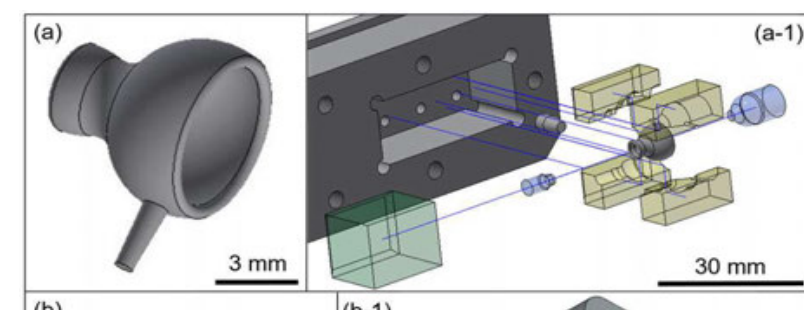

(b)
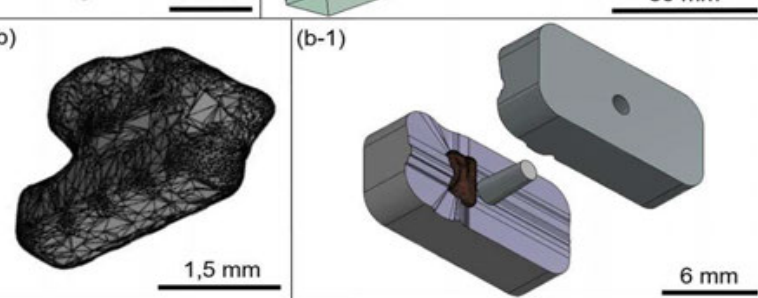

Figure 2:(a) 3D-model of frontal neo-ostium implant prototype with sprue structure; (a-1) scheme of inserting of the mold (7 parts, green, yellow, blue) in metallic housing for $\mu \mathrm{IM}$ process; (b) 3Dmodel of round window niche implant prototype; (b-1) 3Dmodel of RWN-I mold
As material the acrylate-based photopolymer E-Model light from EnvisionTEC was used. E-Model light was not developed for injection molding applications. It has a relatively low heat deflection temperature (HDT) of $60.5^{\circ} \mathrm{C}$ (at $1.82 \mathrm{MPa})$. However, it is a relatively affordable $(220 € / \mathrm{kg})$ and well established DLP 3D printing material for the VIDA device, featuring a relatively low viscosity of $\eta=150 \mathrm{cP}$ (at $\mathrm{T}=30{ }^{\circ} \mathrm{C}$ ) and commonly used for stiff and high-detailed modelling needs. The molds were printed with a xy-resolution of $73 \mu \mathrm{m}$ and a layer height of $50 \mu \mathrm{m}$ (z-resolution) utilising E-Model light specific 3D printing parameters provided from EnvisionTEC. After 3D printing, the single parts of the molds were washed in isopropanol to remove the uncured photopolymer (the cured E-Model light is not soluble in isopropanol). As a next step $10 \mathrm{~min}$ of post-curing was processed using a UV light curing box from EnvisionTEC to finish mold manufacturing.

\subsection{Micro injection molding of implant prototypes}

Implant prototypes were manufactured utilising a $\mu \mathrm{IM}$ device formicaPlast (Klöckner Desma Schuhmaschinen $\mathrm{GmbH}$, Achim, Germany), modified as shown previously [5]. Liquid silicone rubber (LSR) was exemplarily chosen as biocompatible/medically relevant material for implant prototypes manufacturing via $\mu \mathrm{IM}$. For the investigations mold temperatures between $\mathrm{T}_{\text {mold }}=110^{\circ} \mathrm{C}$ up to $\mathrm{T}_{\text {mold }}=160{ }^{\circ} \mathrm{C}$ were used. For the lowest $\mathrm{T}_{\text {mold }}=110{ }^{\circ} \mathrm{C}$ a long curing time of LSR of $t_{\text {curing }} \sim 2 \mathrm{~h}$ is necessary. The highest $\mathrm{T}_{\text {mold }}$ enable much shorter curing times of $t_{\text {curing }} \sim 5 \mathrm{~min}$. In detail $t_{\text {curing }}$ describes the duration of curing of LSR inside the mold after the injecting step and before removing the implant prototypes from the mold. Because of the low HDT of $60.5^{\circ} \mathrm{C}$ of E-Model light probably there is the need for relatively low

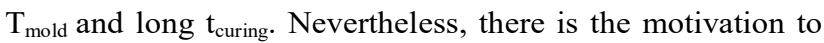
find process parameters that enable as short process time as possible.

\section{Results and discussion}

\subsection{D printing of molds}

Molds for $\mu \mathrm{IM}$ of small-sized RWN-I and FO-I prototypes were 3D printed successfully with layer height of $50 \mu \mathrm{m}$, suitable to be installed in metallic housing for $\mu$ IM (Fig. 3). $3 \mathrm{D}$ printing time was $\sim 20 \mathrm{~min}$ for RWN-I molds and $\sim 60 \mathrm{~min}$ 
for FO-I mold. Because of the small size of the single pieces of one mold, multiple molds can be 3D printed at once (RWNI mold: $\mathrm{n}=20$; FO-I mold: $\mathrm{n}=10$ ). There is at least an additional process time of $\sim 20$ min for manually removing the molds from the $3 \mathrm{D}$ building platform and cleaning with isopropanol as well as another $10 \mathrm{~min}$ for a post-curing process in the UV light curing box. There is a low consumption of the photopolymer E-Model light of $\sim 1.5 \mathrm{~g}$ for a RWN-I mold and $\sim 5.5 \mathrm{~g}$ for a FO-I mold. As a result, we demonstrate a relative time- and cost-saving rapid tooling process for highly detailed, highly complex molds.

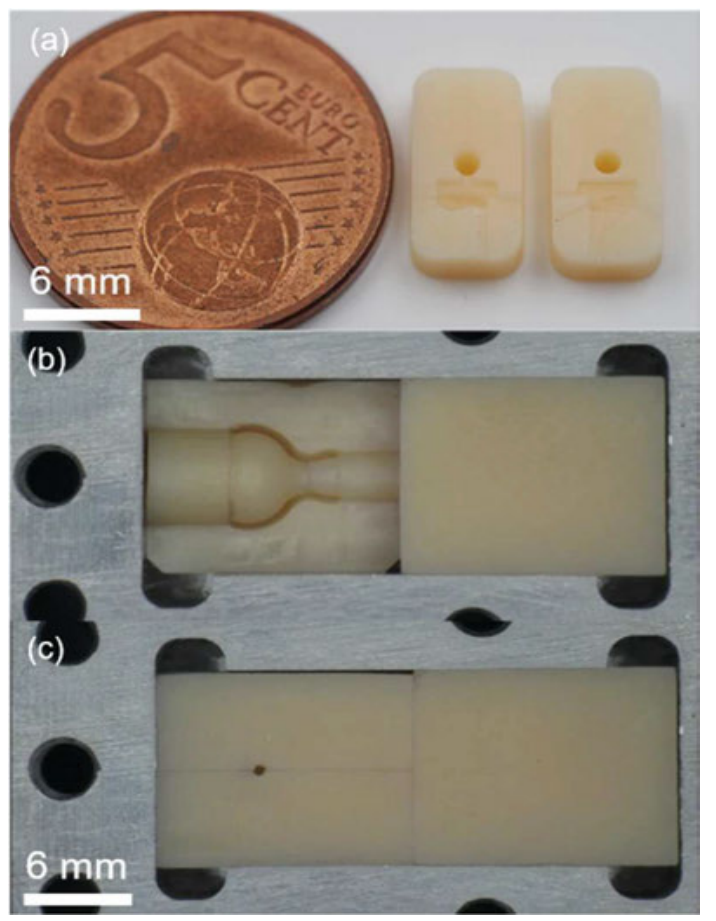

Figure 3: (a) DLP 3D-printed mold for round window niche implant prototype RWN-I; (b) and (c) DLP 3D-printed mold for frontal neo-ostium implant prototype of FO-I mounted in metal mold holder (as planned, Fig. 2 a-1) for micro injection molding $\mu \mathrm{IM}$

\subsection{Micro injection molding of implant prototypes}

The 3D printed molds are sensitive to temperature and show high thermal expansion even at low $\mathrm{T}_{\text {mold }}=110^{\circ} \mathrm{C}$ (Fig. 4 a, b). We found, that instead of decreasing $\mathrm{T}_{\text {mold }}$ to even lower values, what would lead to a inacceptable duration of $t_{\text {curing, }}$ a favourable way for process optimisation is to optimise the design of the metal mold holder. Our results show that a tightfitting insertion of the mold in a metal mold holder enable high $\mathrm{T}_{\text {mold }}=160{ }^{\circ} \mathrm{C}$ (Fig. 4 c). For that purpose it is important to design the mold holder without cavities facing the surface of the mold to prevent thermal expansion of the mold into the cavities. Moreover, we found that the injection pressure must be low and homogeneously distributed. The high thermal expansion of E-Model light molds even helps against burr formation on the moulded parts. Overall, the use of high $\mathrm{T}_{\text {mold }}$ leads to a more reliable process.

Utilising tight supported DLP 3D printed molds, the $\mu \mathrm{IM}$ of RWN-I and FO-I prototypes using $\mathrm{T}_{\text {mold }}=160{ }^{\circ} \mathrm{C}$ and $\mathrm{t}_{\text {curing }}=5$ min were successful (Fig. 4 d, Fig. 5). We used a mold for up to $\mathrm{n}=8 \mu \mathrm{IM}$ cycles in a row and no significant

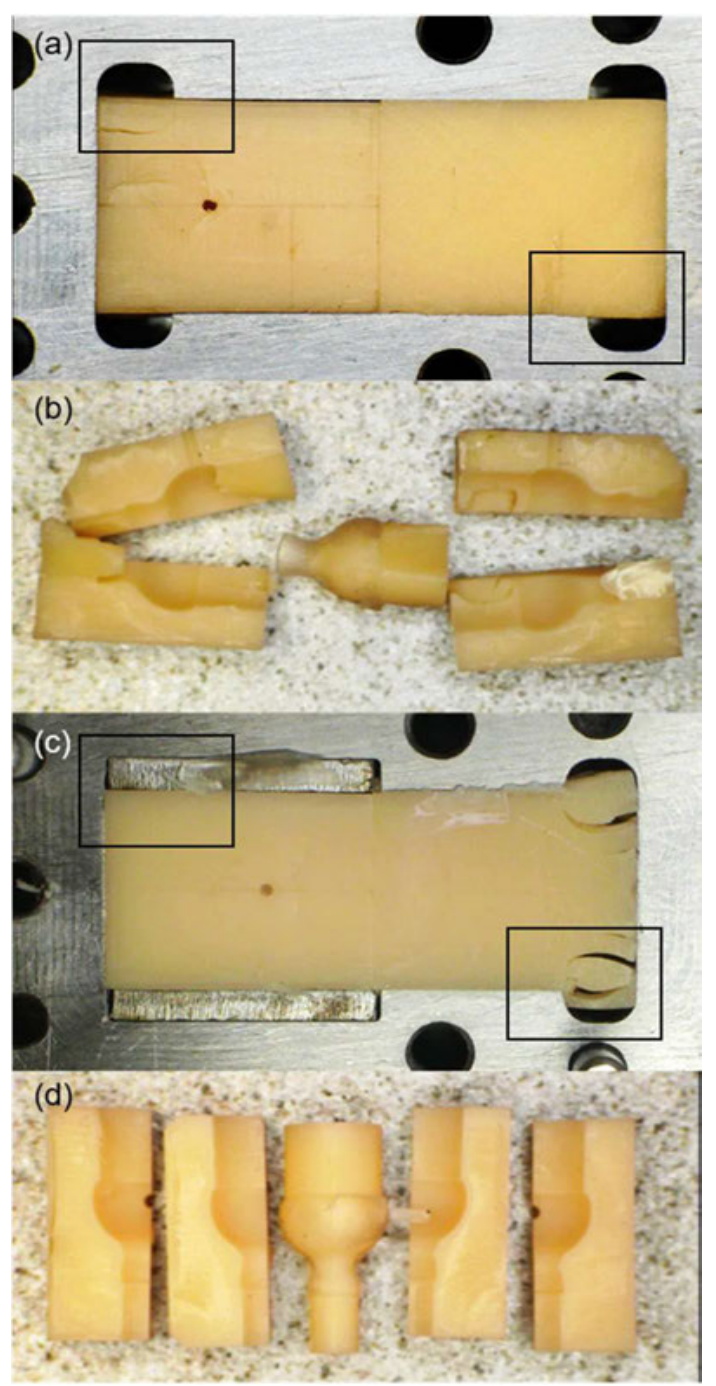

Figure 4: (a) mold after utilizing low $T_{\text {mold }}=110^{\circ} \mathrm{C}$. Bulging and cracking after thermal stress can be seen especially in handling-cavities (for removing) of metal mold holder. (b) $\mu \mathrm{IM}$ manufactured FO-I shows deforming because of cracked mold parts. (c) mold after utilising high $\mathrm{T}_{\text {mold }}=160^{\circ} \mathrm{C}$. Because of optimised design of metal mold holder (without cavities), there is no cracking or deforming on that cavity-less part of the mold (left). Strong deforming and cracking is shown in other part of the mold (right), where the mold holder features cavities (for inserting/removing of mold). (d) no significant deforming and cracking of mold parts and implant protoype when optimised mold holder design is used 
quality loss of implant prototypes since no deforming or cracks were determined. The surface of implant prototypes shows a staircase-effect (Fig. 5). This is a consequence of manufacturing the molds via a $3 \mathrm{D}$ printing process. Nevertheless, because of the relatively high DLP 3D printing resolution and the small layer height of $50 \mu \mathrm{m}$, the implant prototypes were manufactured with adequate accuracy via $\mu \mathrm{IM}$.

For $\mu \mathrm{IM}$ rapid tooling applications, the use of $3 \mathrm{D}$ printing materials featuring a higher $\operatorname{HDT}\left(>95^{\circ} \mathrm{C}\right)$ is common [4]. However, when there is a suitable tight-fitting metallic mold holder for the 3D printed molds, the use of low HDT 3D printing materials is possible, as demonstrated here for the photopolymer E-Model light, which features relatively high 3D-printing resolution. Moreover, the thermal expansion of the material helps to prevent burr formation. Small gaps between the single pieces of a FO-I mold will be sealed during the $\mu \mathrm{IM}$ process because of the thermal expansion of E-Model light.

\section{Conclusion}

In conclusion, we present a $\mu \mathrm{IM}$ process, which is promising for the manufacturing of small-sized, patient-individualised, drug-eluting implants for round window niche and for frontal sinus drainage. Our manufacturing process combines the advantages of DLP, which enables time- and cost-savings, high-precision rapid tooling of molds, and the benefit of $\mu \mathrm{IM}$, which allows the processing of a wide variety of polymeric materials for the manufacturing of small-sized, complexshaped implants. We demonstrate that an acrylate-based photopolymer DLP material with a low $\mathrm{HDT}=60.5^{\circ} \mathrm{C}$ is suitable for our process, though there is the need for adequate design of a (metal) mold holder. There is the need for perfect fit without cavities facing the surface of the mold so as to prevent cracking and deforming because of thermal expansion into the cavities.

Further work will focus on the investigation of $\mu \mathrm{IM}$ parameters for other (bio)medical relevant materials such as biodegradable polymers as well as drug-laden polymers.

\section{Author Statement}

The authors would like to thank the Federal Ministry of Education and Research of Germany (BMBF) for research funding of RESPONSE-Partnership for Innovation in Implant Technology in the program Zwanzig20 - Partnership for Innovation. The authors state no conflict of interest.

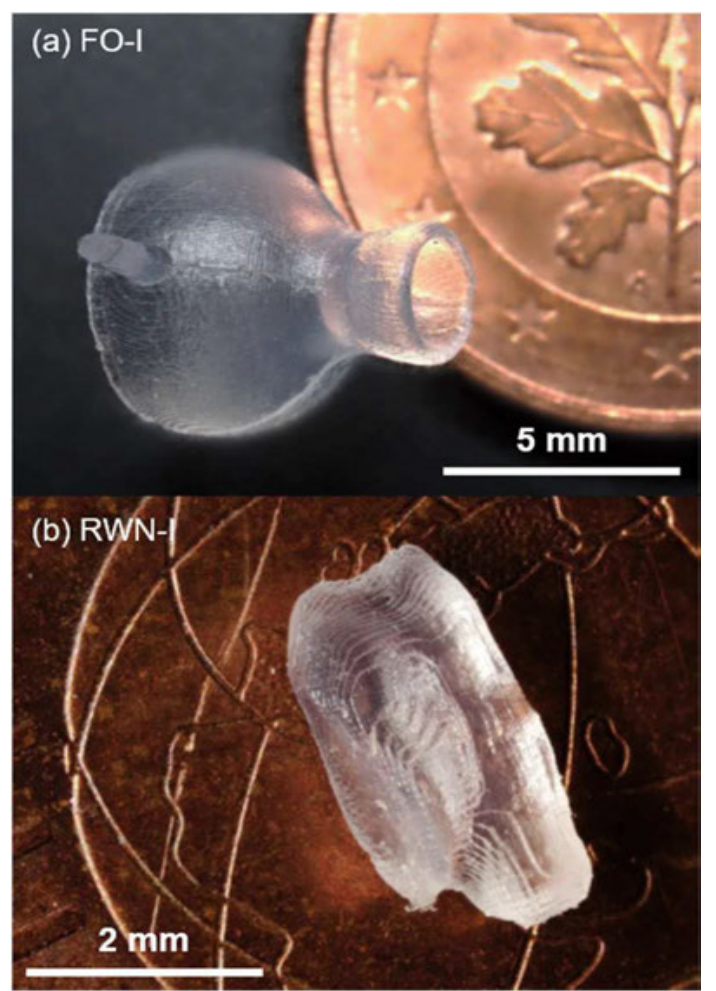

Figure 5: (a) Finished FO-I prototype and (b) finished RWN-I prototype. Both are manufactured from LSR. High accuracy, no cracks or deforming. The surfaces of both prototypes show a staircase-effect, caused by rapid tooling of molds via 3D printing.

\section{References}

[1] Matin F et al. (2021) Determination of the Round Window Niche Anatomy Using Cone Beam Computed Tomography Imaging as Preparatory Work for Individualized DrugReleasing Implants. J Imaging 7:79. https://doi.org/10.3390/jimaging7050079

[2] Gao Z et al. (2020) High Variability of Postsurgical Anatomy Supports the Need for Individualised Drug-Eluting Implants to Treat Chronic Rhinosinusitis. Life (Basel) 10. https://doi.org/10.3390/life10120353

[3] Calaon M et al. (2020) Functional Analysis Validation of Micro and Conventional Injection Molding Machines Performances Based on Process Precision and Accuracy for Micro Manufacturing. Micromachines (Basel) 11. https://doi.org/10.3390/mi11121115

[4] Dempsey D et al. (2020) Characterisation of Stereolithography Printed Soft Tooling for Micro Injection Molding. Micromachines (Basel) 11. https://doi.org/10.3390/mi11090819

[5] Jüttner $\mathrm{G}$ et al (2011) Tailor made micro parts in small series and mass production. In: Tagungsband der 45. DGBMT Jahrestagung, 3-Länder-Tagung D-A-CH, (BMT2011), Freiburg i. Br. 2011, P89/Track I. 